\title{
Traumatic Axonal Injury in the Spinal Cord Evoked by Traumatic Brain Injury
}

\author{
ENDRE CZEITER, ${ }^{1}$ JOZSEF PAL, ${ }^{1}$ ERZSEBET KOVESDI, ${ }^{1}$ PETER BUKOVICS,,${ }^{1,2}$ \\ JANOS LUCKL, ${ }^{1}$ TAMAS DOCZI, ${ }^{1}$ and ANDRAS BUKI ${ }^{1}$
}

\begin{abstract}
Although it is well known that traumatic brain injury (TBI) evokes traumatic axonal injury (TAI) within the brain, TBI-induced axonal damage in the spinal cord (SC) has been less extensively investigated. Detection of such axonal injury in the spinal cord would further the complexity of TBI while also challenging some functional neurobehavioral endpoints frequently used to assess recovery in various models of TBI. To assess TAI in the spinal cord associated with TBI, we analyzed the craniocervical junction (CCJ), cervico-thoracic (CT), and thoraco-lumber (ThL) spinal cord in a rodent model of impact acceleration of TBI of varying severities. Rats were transcardially fixed with aldehydes at 2,6 , and $24 \mathrm{~h}$ post-injury $(n=36)$; each group included on sham-injured rodent. Semi-serial vibratome sections were reacted with antibodies targeting TAI via alteration in cytoskeletal integrity or impaired axonal transport. Consistent with previous observations in this model, the CCJ contained numerous injured axons. Immunoreactive, damaged axonal profiles were also detected as caudal, as the ThL spinal cord displayed morphological characteristics entirely consistent with those described in the brainstem and the CCJ. Quantitative analyses demonstrated that the occurrence and extent of TAI is positively associated with the impact/energy of injury and negatively with the distance from the brainstem. These observations show that TBI can evoke TAI in regions remote from the injury site, including the spinal cord itself. This finding is relevant to shaken baby syndrome as well as during the analysis of data in functional recovery in various models of TBI.
\end{abstract}

Key words: animal studies; axonal injury; models of injury; traumatic brain injury

\section{INTRODUCTION}

$\mathbf{T}$ RAUMATIC BRAIN INJURY (TBI) evokes widespread/ diffuse axonal injury (DAI), which contributes to its morbidity and mortality (Adams et al., 1991). Our current understanding recognizes DAI to be a progressive process evoked by the tear/shearing forces of accelera- tion-deceleration injury. These forceps can cause axonal alterations leading to ultimate disconnection-either via the formation of swollen axon bulbs or swelling, vacuolization, and disruption of the injured axon-segment (Povlishock and Stone, 2001; Povlishock et al., 1983; Povlishock, 1993). In humans, such brain injuries evoke DAI in the corpus callosum, subcortical white matter, and

\footnotetext{
${ }^{1}$ Department of Neurosurgery, University of Pécs, Pécs, Hungary.

${ }^{2}$ Clinical Neuroscience Research Group, Hungarian Academy of Sciences, Pécs, Hungary.
} 


\section{CZEITER ET AL.}

the brainstem-typically resulting in a deeply altered level of consciousness. This may occur in the absence of any space-occupying lesion on conventional computed tomography (CT) scans (Adams et al., 1991; Blumbergs, 2005; Gennarelli et al., 1982).

In rodents, this pathology is replicated in models of impact acceleration (weight-drop) head injury, where rapid flexion-extension of the head and in the craniocervical junction (CCJ) leads to shearing of long fiber tracts (Marmarou et al., 1994; Povlishock et al., 1997). In experimental models, DAI is frequently referred to as traumatic axonal injury (TAI) (Maxwell et al., 1997). Although on the basis of the distribution of the energy/ forces evoking TAI, it is reasonable to assume the potential occurrence of spinal cord (SC) damage, to date only limited information has been collected on this issue, with limited descriptions of axonal injury in the cervical segments in controlled cortical impact (CCI) (Lighthall, 1988) and rotational acceleration injury (Hamberger et al., 2003). In humans, only anecdotal data are available regarding the occurrence of DAI in the cervical spinal cord, primarily in shaken baby syndrome (SBS) (Shannon et al., 1998).

The present study was initiated to investigate the occurrence of TAI in the CCJ and, in an attempt to define the extent of such TAI, the cervico-thoracic (CT) and the thoraco-lumbar (ThL) SC in a rat model of impact acceleration TBI. Widely used immunohistochemical (IC) markers of TAI-beta amyloid precursor protein (APP) and RMO-14 - were utilized to demonstrate and quantitatively analyze axonal damage. The RMO-14 antibody is known to exclusively target an epitope on the neurofilament rod domains of altered neurofilament $M$ subunits (NFM), which are exposed upon modification of the NF sidearms, an assumed consequence of calcium induced enzymatic processes evoked by TBI (Lee et al., 1987), whereas APP carried by fast axonal transport is pooled at foci affected by the injury (Sherriff et al., 1994a,b).

\section{METHODS}

The well-characterized rodent model of impact acceleration TBI was utilized as described before (Marmarou et al., 1994). In all, 36 Wistar rats (Charles River Hungary) (385-405 g) were used for the experiments, including six animals serving as sham-injured controls. For the induction of anesthesia, the animals were exposed to $4 \%$ isoflurane (Forane, Abbott, Hungary) in a bell jar for $5 \mathrm{~min}$, and then intubated and ventilated (Inspira Advanced Safety Ventillator, Harvard Apparatus) with a mixture of $1-2 \%$ isoflurane in $30 \% \mathrm{O}_{2}$ and $70 \% \mathrm{~N}_{2} \mathrm{O}$. One group of the animals $(n=15)$ underwent mild TBI (inflicted with brass weights of $450 \mathrm{~g}$ from a height of $100 \mathrm{~cm}$ according to previous descriptions), while the rest of the rats $(n=15)$ were exposed to moderate/severe impact acceleration TBI (inflicted with brass weights of 450 $\mathrm{g}$ from a height of $200 \mathrm{~cm}$ ). Following the injury, rats were ventilated with $100 \% \quad \mathrm{O}_{2}$ until regaining consciousness. The respiratory status (pulse oximetry: Engström EOS [Sweden]), temporalis muscle, and rectal temperature as well as non-invasive blood pressure was monitored (Temperature Control/Heating Pad, Bowdoinham [Physitemp/model BAT-12]). At the pre-determined survival of 2,6 , and $24 \mathrm{~h}$ after injury $(n=5+1$ sham/ group), rats were transcardially fixed with aldehydes.

The body temperature was maintained at normothermic levels.

At the designated survival time, rats were re-anesthetized with an overdose of sodium pentobarbital and transcardially perfused with $4 \%$ paraformaldehyde in phosphate-buffered saline (PBS).

Brains along with the SC were removed and immersed in the same fixative overnight (16-18 h). On the basis of previously published observations concerning the topography of TAI in rat brain (Buki et al., 1999; Koizumi and Povlishock, 1998) a midline, 5-min-wide block of the brain was removed using a sagittal brain blocking device to include the region extending from the interpeduncular fossa to the second cervical segment (World Precision Instruments, Sarasota, FL). Similarly, the entire SC has been removed and blocked transversally to provide tissue blocks from the CTh and the ThL SC. All blocks were sectioned sagittally with Vibratome Series 1000 (Polysciences Inc., Warrington, PA) at a thickness of 30 $\mu \mathrm{m}$ and collected in $0.1 \mathrm{M}$ phosphate buffer. Sections were collected in a semi-serial fashion in two groups; the first group was processed for immunohistochemical (IC) localization of RMO-14, the second for APP respectively. Having been washed with PBS for three 10-min periods, after the suppression of endogenous peroxidase activity by a 30 -min treatment with $0.5 \%$ hydrogen peroxide (Reanal, Hungary) in PBS followed by washing three times in PBS, the sections were exposed to controlled-temperature microwave antigene-retrieval in citrate buffer (6 pH) with PELCo BioWave 34700-230 (Stone et al., 1999). After three quick rinses in PBS, sections were immersed for $40 \mathrm{~min}$ in $1 \%$ normal horse serum (NHS) (Vectastain Universal Elite ABC Kit, Vector PK-6200) diluted with PBS (1\% NHS/PBS), incubated overnight in rabbit anti-APP antibody (Zymed Laboratories, Inc., cat. no. 51-2700) diluted with $1 \%$ NHS/PBS at $1: 250$, or in mouse monoclonal RMO14 antibody at a dilution of 1:500 (kindly provided by Dr. John Q. Trojanowski, University of Pennsylvania Department of Pathology) and then washed with PBS three times for $10 \mathrm{~min}$. Thereafter, 
the sections were subjected to the staining protocol of the Vectastain Universal Elite ABC Kit (Vector, cat. no. PK6200). Finally, the endproduct of the immunohistochemical reaction was visualized with a diaminobenzidine (DAB) peroxidase substrate kit (Vector, cat. no. SK4100) and then washed with PBS for $10 \mathrm{~min}$. Next the sections were mounted and cleared for routine light microscopic examination.

The employed antibodies have been well characterized and widely used. However, to further assure specificity all IC incuations included sections processed without the addition of the primary or secondary antibodies.

Image analysis of selected sections was performed according to our previously employed protocol (Buki et al., 2000).

At the craniocervical junction semi-serial sections (six from each animal/each antibody) were examined with a NIKON 600 light microscope equipped with a SPOT Diagnostic Instruments Inc. SPOT-RT 2.2.1 digital camera interfaced with a computer-assisted image analysis system (utilizing NIH Image $\mathrm{J}$ and UTHSCSA-Imagetool 3.00 ) in a blinded fashion. At the cervicomedullary junction two adjacent grids of $40,000 \mu \mathrm{m}^{2}$ were superimposed over the corticospoinal tracts (CSpT), images were captured and digitized at a magnification of $50 \times$, and the total number of damaged APP or RMO14 immunopositive axonal profiles within this area were marked and counted. In the same brainstem sections an adjacent image of the medial longitudinal fasciculus (MLF) was captured and digitized at $25 \times$, and a $160,000 \mu \mathrm{m}^{2}$ grid was superimposed over the region of the MLF at the medulla and adjacent $\mathrm{C} 1$ segment and the number of damaged axonal profiles within the grid was counted and expressed as density of APP or RMO-14-immunopositive axons.

At the cervico-thoracic junction, six semi-serial sections from each animal have been selected, and all damaged immunoreactive profiles were counted over a diameter of $2 \mu \mathrm{m}$ within the area of the above-described grid adjusted to the region where the bulk of damaged axons appeared. The number of damaged axonal profiles within the grid was counted and expressed as density of APP-immunopositive or RMO-14-immunopositive axons.

At the thoraco-lumbar junction, due to the relatively low number of immunoreactive (IR) axonal segments, absolute number of damaged, immunoreactive axonal profiles from six sections per animal were used for statistical analysis.

\section{Statistical Analysis}

Two-way analysis of variance (ANOVA) was used to compare the density of immunopositive profiles (expressed as number $/ \mathrm{mm}^{2}$; or, alternatively, in the case of the ThL region, the absolute number of damaged immunoreactive axons) between various groups of animals. Differences were assumed significant at a level of $p \leq 0.05$. Significance was clarified by Scheffe post hoc test where required.

\section{RESULTS}

\section{Physiological Findings}

In accordance with previous observations (Buki et al., 1999), the use of the above-described experimental protocols did not result in any significant alteration in the physiological parameters monitored.

\section{Light Microscopic Examination}

Sham-injured animals did not display immunoreactive (IR) axonal profiles. In contrast, animals subjected to TBI and reacted for the visualization of the above characterized antibodies showed discrete IC reaction products within scattered axons predominately localized in the corticospinal tract and medial longitudinal fasiculus in the brainstem. Morphological characteristics of the swollen, occasionally disconnected APP-IR-axonal segments and the lobulated/vacuolated, partially or totally disconnected RMO-14 IR-axonal segments were entirely consistent with previous descriptions of TAI 2-24 h post-injury (Povlishock and Stone, 2001; Stone et al., 1999).

In the CCJ damaged, IR-axonal segments were primarily localized in the corticospinal tract. In the CTh and the ThL SC damaged, immunoreactive axons occupied the dorsal columns and occasionally were found in the ventral white matter.

Morphological characteristics as well as IR of these axonal profiles were consistent with those described in the brainstem (Figs. 1 and 2). The majority of the axons were RMO-14 positive, displaying vacuolization at $2 \mathrm{~h}$ and disconnection as well as fragmentation at $24 \mathrm{~h}$ postinjury. Of interest was the observation that APP-IR axons in the CTh and ThL segments appeared similar to the RMO-14 immunoreactive segments (Fig. 3).

Qualitative observations suggested that IR, damaged axons were detected less frequently in animals sustained mild injury, and in the ThL region of these animals no immunoreactive axonal profiles were observed.

Overall, the density of damaged IR axonal profiles as well as the proportion of smaller caliber axons decreased dramatically from cranial to SC caudal segments.

\section{Quantitative Analysis}

Quantitative analysis of brain tissue reacted with either APP or RMO-14 antibody confirmed our qualita- 


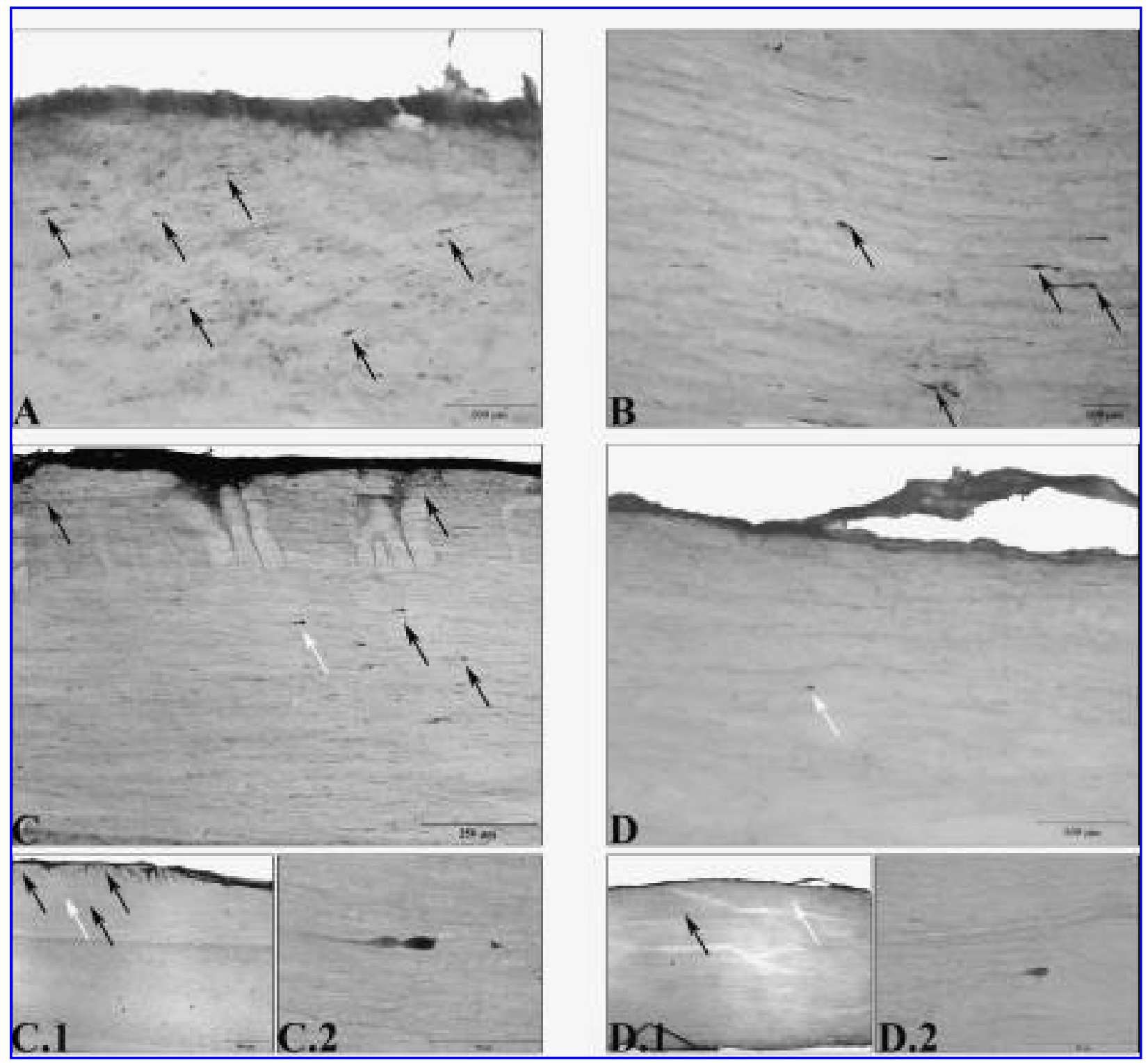

FIG. 1. Traumatically injured axons displaying amyloid precursor protein (APP) immunoreactivity in the craniocervical junction primarily in the corticospinal tracts (CSpT; A) and the medial longitudinal fasciculus (MLF) (B). Arrows indicate swollen axonal profiles. There are scattered APP-immunoreactives (IRs) in the cervical and cervicothoracic region (C, C.1, C.2, where C was enlarged from C.1 and C.2 was enlarged from C) displaying focal swelling and imminent disconnections (C.2). Light micrographs of the thoraco-lumbar region (D, D.1, D.2, where D was enlarged from D.1 and D.2 was enlarged from D) demonstrating APP-IR axon-segments. White arrows point at the same APP-IR axon. All images represent APP-IRs at $6 \mathrm{~h}$ post-injury.

tive observations described at the craniocervical junction and in the brainstem (Table 1): the number of damaged, IR axonal segments correlated significantly with the force of impact with two-way ANOVA between 1 and $2 \mathrm{~m}$ at either 2, 6, or $24 \mathrm{~h}$ post-injury both in CSpT or in MLF ( $p$ values in the CSpT were $<0.001$ with APP and RMO-14 labeling and $p$ values in the MLF were also $<0.001$ either with APP or RMO-14 labeling). The number of immunoreactive axonal segments significantly increased between 2 and 6 h post-injury: $p<0.001$ in CSpT with APP labeling, while no significant alteration between 6 and 24 h postinjury was observed (two-way ANOVA with a Scheffe post hoc test). In the severe head injury group ( $2 \mathrm{~m}$ impact), the number of damaged RMO-14 immunoreactive axons proved significantly higher at all time points than that of the APP-IR ones, particularly in the MLF $(p<0.001$ with two-way ANOVA) (Fig. 4). 


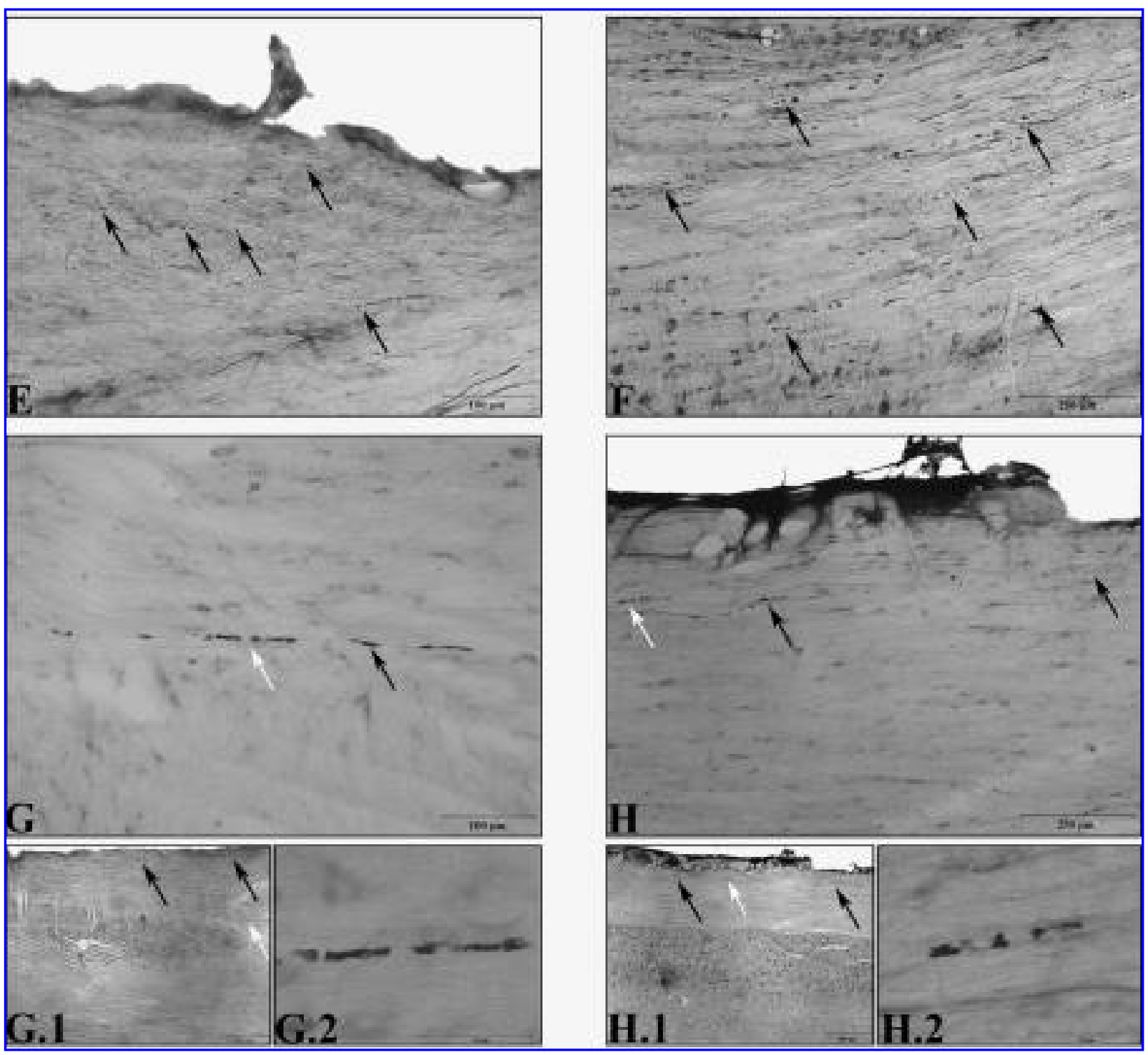

FIG. 2. Representative RMO-14 immunostained sections of the craniocervical junction displaying the corticospinal tracts (CSpT; E) and the FLM (F). Arrows indicate immunoreactive (IR) axons in the CSpT and wide caliber axon profiles in the MLF. Characteristic vacuolated damaged axons were identified in the cervical and cervicothoracic region (G, G.1, G.2, where G was enlarged from G.1 and G.2 was enlarged from G) and in the thoraco-lumbar region (H, H.1, H.2, where H was enlarged from H.1 and H.2 was enlarged from H) demonstrating RMO-14-IR axon segments. White arrows point at the same RMO-14-IR axon. All images represent RMO-14-IRs at $6 \mathrm{~h}$ post-injury.

Similar observations were made on the second, cervicothoracic block. In this group, the axonal number plateaued at $6 \mathrm{~h}$ post-injury $(p=0.006$ between 2 and $6 \mathrm{~h}$ with APP labeling (two way ANOVA with a Scheffe post hoc test), where the relation between axonal injury and the impact was even more striking $(p<0.001$ between 1 and $2 \mathrm{~m}$ impact with APP and $p<0.001$ with RMO-14 labeling. Similar to the above detailed observations made in the CCJ, the number of RMO-14 immunopositive axons ap- peared significantly higher than the number of APP-positive axons, particularly in the severe head injury group $(p<$ 0.001 with two-way ANOVA) (Fig. 4).

In the ThL region, virtually no immunoreactivity was observed on the mild head injury group. The absolute number of RMO-14 IR axonal segments was again significantly higher in the APP ones $(p<0.001$ two-way ANOVA), with both RMO-14 and APP reaching maximum levels at $6 \mathrm{~h}$ post-injury (Fig. 4). 


\section{CZEITER ET AL.}

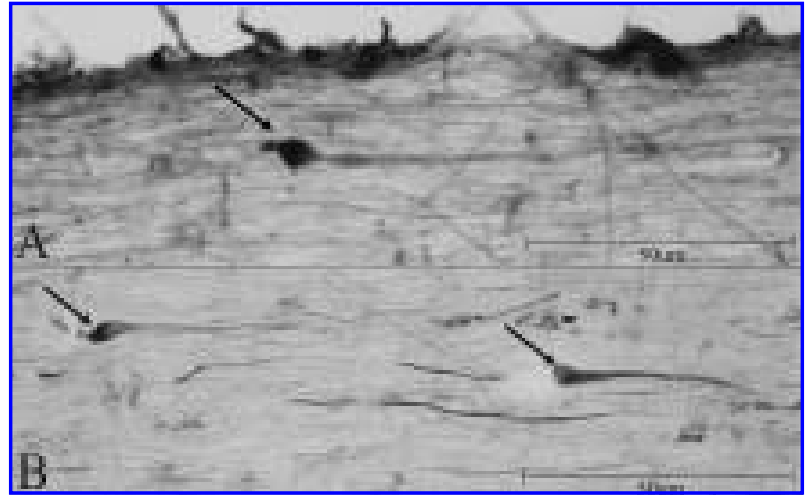

FIG. 3. One amyloid precursor protein-immunoreactive (APP-IR) (A) and two RMO-14-IR (B) axon-segments from the area of the cervicothoracic block $2 \mathrm{~h}$ post-injury (arrows). RMO-14 and APP-IR appear strikingly similar.

\section{DISCUSSION}

In the present study, we demonstrate using a rodent model of impact acceleration TBI which is widely used to analyze diffuse brain injury that this model is also capable of evoking TAI in virtually all segments of the SC. Injured axons are most frequently located in the $\mathrm{CCJ}$ and the CTh region and the relative amount of RMO-14-IR seems to predominate over the APP-IR segments, particularly in the ThL SC.

The impact acceleration model of TBI described by Marmarou et al. (1994) is a widely used experimental model to evoke TAI in rodents. This model is capable of producing TAI without significant focal damage such as contusional change. Further, the model is both reliable and reproducible. Although this model has been well characterized both by original descriptions and further studies, the potential for axonal injury in the spinal cord was not previously evaluated (Marmarou et al., 1994; Maxwell et al., 1997).

Generation of axonal injury in the SC can be potentially explained by several theories. First, it has been suggested that axonal injury in this model is evoked by rapid flexion-extension at the CCJ. On this basis, it is likely that stretching of the long fiber tracts also generates axonal injury at remote sites. Secondly, the shock wave triggered by the traumatic injury could advance through the cerebrospinal fluid at the moment of injury to contribute to remote axonal injury. It is of note that the majority of injured axons in the current investigation particularly in the ThL region were seen close to the surface of the spinal cord. Interestingly, preliminary unpublished observations suggest that with central fluid percussion brain injury damaged axonal segments can also be detected in the spinal cord pointing to the potential etiological role of shock wave pressure gradient triggered by the initial impact.

Further, as the spinal cord is attached to the adjacent dura via the dentate ligaments and also anchored to the entering roots, acceleration-deceleration injuries would seem capable of evoking structural damage at virtually all segments of the cord.

As recent studies suggest that Wallerian degeneration may occur in the CNS earlier than previously postulated, the possibility that some of the RMO-14 IR detected might be linked to axotomy occurring in the brainstem or the rostral spinal cord, cannot be excluded (Kelley et

Table 1. Results of the Quantitative Data Analysis Summarizing the Densities of Damaged APP-IR and RMO-14-IR Axonal Profiles in the Craniocervical Junction as well as in the Cervico-Thoracic Region and Absolute Number of Damaged Axons in the Thoraco-Lumbar Block

\begin{tabular}{|c|c|c|c|c|c|c|}
\hline \multirow[b]{2}{*}{ Block } & \multirow[b]{2}{*}{ Region } & \multirow{2}{*}{$\begin{array}{c}\text { Survival } \\
\text { time } \\
\text { (h) }\end{array}$} & \multicolumn{2}{|c|}{$A P P$, mean $\pm S D$} & \multicolumn{2}{|c|}{$R M O-14$, mean $\pm S D$} \\
\hline & & & $1 m$ & $2 m$ & $1 m$ & $2 m$ \\
\hline \multirow{6}{*}{$\begin{array}{l}\text { 1. Craniocervical } \\
\text { junction }\end{array}$} & \multirow[t]{3}{*}{ CSpT } & 2 & $188 \pm 30.3$ & $542 \pm 48.2$ & $104 \pm 51.2$ & $984 \pm 68.9$ \\
\hline & & 6 & $260 \pm 36.7$ & $862 \pm 37.7$ & $118 \pm 19.4$ & $1114 \pm 255.2$ \\
\hline & & 24 & $306 \pm 91.3$ & $888 \pm 101.3$ & $116 \pm 48.4$ & $1100 \pm 374.2$ \\
\hline & \multirow[t]{3}{*}{ FLM } & 2 & $104 \pm 20.7$ & $228 \pm 40.9$ & $54 \pm 28.7$ & $436 \pm 40.3$ \\
\hline & & 6 & $154 \pm 32.1$ & $256 \pm 67.3$ & $66 \pm 33.8$ & $494 \pm 60.2$ \\
\hline & & 24 & $124 \pm 57.7$ & $254 \pm 68.0$ & $46 \pm 27.3$ & $466 \pm 69.7$ \\
\hline \multirow[t]{3}{*}{ 2. Cervicothoracic } & & 2 & $22 \pm 14.8$ & $96 \pm 20.7$ & $44 \pm 27.3$ & $204 \pm 37.2$ \\
\hline & & 6 & $54 \pm 11.4$ & $154 \pm 45.1$ & $46 \pm 28.7$ & $256 \pm 84.5$ \\
\hline & & 24 & $26 \pm 24.1$ & $116 \pm 15.2$ & $30 \pm 16.7$ & $222 \pm 41.2$ \\
\hline \multirow{3}{*}{$\begin{array}{l}\text { 3. Thoracolumbar } \\
\text { (absolute amount) }\end{array}$} & & 2 & Neg & $7.6 \pm 3.8$ & Neg & $17.6 \pm 5.1$ \\
\hline & & 6 & $\mathrm{Neg}$ & $7.6 \pm 4.1$ & $\mathrm{Neg}$ & $20.8 \pm 5.3$ \\
\hline & & 24 & $\mathrm{Neg}$ & $5.6 \pm 2.4$ & $\mathrm{Neg}$ & $14.8 \pm 3.4$ \\
\hline
\end{tabular}

SD, standard deviation; CSpT, corticospinal tracts; MLF, medial longitudinal fasciculus; neg, negligible. 


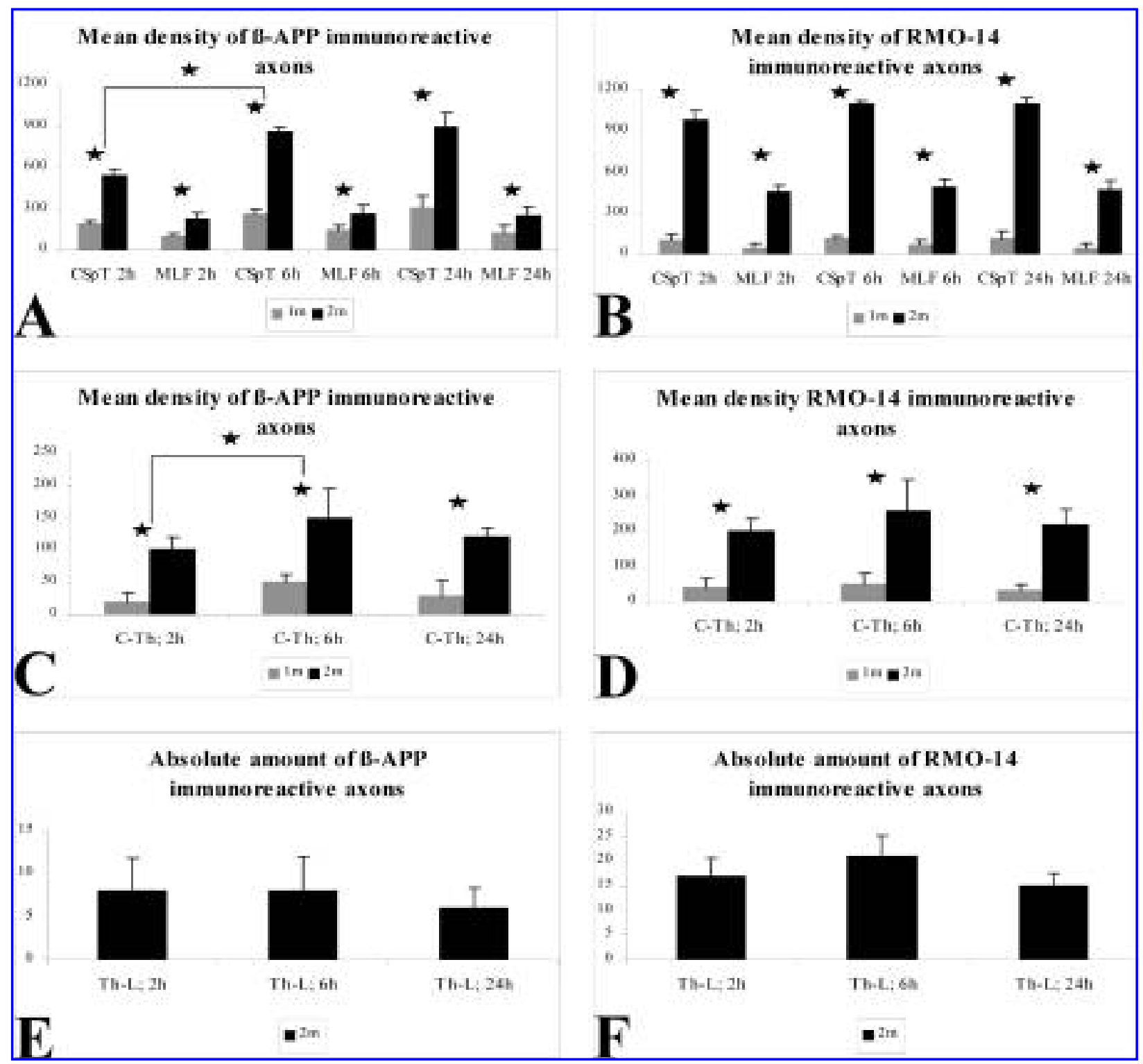

FIG. 4. These diagrams compare the amount of amyloid precursor protein-immunoreactive (APP-IR) and RMO-14-IR axonsegments in the various blocks of the spinal cord, defined in the text. In the craniocervical junction, the mean densities of APPIR (A) and RMO-14-IR (B) axonal segments similar to several previous descriptions. In the cervicothoracic block, lower density values were found for both APP-immunopositive $(\mathbf{C})$ and RMO-14-immunopositive (D) profiles, but in the case of severe head injury (2-m impact) there is still quite a large number of damaged axons in the thoraco-lumbar block as far as the absolute number of APP-IR (E) and RMO-14-IR (F) axonal segments is considered. Asterisks indicate the statistically significant differences.

al., 2006). Nevertheless, neither the detection of APP-IR nor the magnitude of axonal damage at two hour post injury could be explained by secondary axonal degeneration alone.

Several studies have addressed the issue of the heterogeneity of axonal injury in terms of axon caliber and ICC markers of TAI (Povlishock and Stone, 2001). Although the work presented here did not employ double labeling strategies, comparison of the morphological characteristics of the damaged axons indicate that primarily large caliber axons are affected in the spinal cord similar to those that were previously described in the MLF displaying RMO-14-IR. While there is a considerable debate, whether these large-caliber axons display APP-IR, and, if they do, when does this APP-IR first occur post-injury, the RMO-14 and APP-IR axons appear 
strikingly similar as early as $2 \mathrm{~h}$ post-injury (Fig. 3). It is also of note that RMO-14 IR is detected in large axonal swellings indicative of axonal disconnection at this early time point.

Although this issue requires further analysis via double-labeling ICC strategies, two points are of note. First, as was postulated previously, APP-IR alone seriously underestimates the extent of axonal damage. Thus, routine forensic studies should not rely exclusively on this modality. Second, large calbier axons in the spinal cord appear to display cytosketal alterations with concomitant impaired axonal transport earlier post-injury than described in the brainstem.

Our finding that mild TBI wherein virtually no neurological dysfunction has been noted in rodents also evokes TAI in remote regions of the SC suggests that axonal injury may occur in the SC of patients who have suffered acceleration-deceleration type injury or whiplash injury without overt clinical signs of any SC damage. In light of this premise, the significance of repeated micro-injuries particularly in professional athletes may require renewed attention. Further, repeated minor injuries could be of additional importance in elderly patients, where shearing of the spinal cord on the surface of spondylotic spurs in extension-axial compression trauma could contribute to the development of spondylotic myelopathy, a condition recently now more frequently associated with the occurrence of axonal damage besides the classical pathogenetic role of vascular compromise.

Additionally, our obsevations are further supported by Cornish et al. (2000), who have described axonal injury in SC segments remote to the site of severe spinal cord injury.

Our findings also provide indirect support of previous observations that have described TAI in the CCJ in SBS, suggesting a more consistent analysis of the $\mathrm{SC}$ via various IC-markers in all purported cases of SBS.

\section{CONCLUSION}

Our observations demonstrate that acceleration-deceleration type TBI evokes widesperad TAI also involving remote regions of the SC. While these findings may be of relevance to human TBI, in animal investigations of TBI the concomitant SC damage should also be considered on the evaluation of functional recovery. Further IC double/multiple labeling- and immuno-electromicroscopic studies should provide additional information on the pathobiology of TAI in the SC comparing its ultrastructural features with those well described characteristics of TAI in the cerebrum and the brainstem.

\section{ACKNOWLEDGMENTS}

FIRCA (1-RO3-TW0131302A1), NIH (grant NS20193), National Science Projects (NKFP1A/00026/2002 and ALK 000126/2002), Békési Scholarship, and OTKA (T048724-OPR) supported this work.

\section{REFERENCES}

Adams, J.H., Graham, D.I., Gennarelli, T.A., and Maxwell, W.L. (1991). Diffuse axonal injury in non-missile head injury. J. Neurol. Neurosurg. Psychiatry 54, 481-483.

Blumbergs, P.C. (2005). Pathology, in: Head injury. P.L. Reilly and R. Bullock (eds), Hodder Arnold: London, pps. 41-72.

Buki, A., Siman, R., Trojanowski, J.Q., and Povlishock, J.T. (1999). The role of calpain-mediated spectrin proteolysis in traumatically induced axonal injury. J. Neuropathol. Exp. Neurol. 58, 365-375.

Buki, A., Okonkwo, D.O., Wang, K.K., and Povlishock, J.T. (2000). Cytochrome c release and caspase activation in traumatic axonal injury. J. Neurosci. 20, 2825-2834.

Cornish, R., Blumbergs, P.C., Manavis, J., Scott, G., Jones, N.R., and Reilly, P.L. (2000). Topography and severity of axonal injury in human spinal cord trauma using amyloid precursor protein as a marker of axonal injury. Spine 25, 1227-1233.

Gennarelli, T.A., Thibault, L.E., Adams, J.H., Graham, D.I., Thompson, C.J., and Marcincin, R.P. (1982). Diffuse axonal injury and traumatic coma in the primate. Ann. Neurol. 12, 564-574.

Hamberger, A., Huang, Y.L., Zhu, H., et al. (2003). Redistribution of neurofilaments and accumulation of beta-amyloid protein after brain injury by rotational acceleration of the head. J. Neurotrauma 20, 169-178.

Kelley, B.J., Farkas, O., Lifshitz, J., and Povlishock, J.T. (2006). Traumatic axonal injury in the perisomatic domain triggers ultrarapid secondary axotomy and Wallerian degeneration. Exp. Neurol. 198, 350-360.

Koizumi, H., and Povlishock, J.T. (1998). Posttraumatic hypothermia in the treatment of axonal damage in an animal model of traumatic axonal injury. J. Neurosurg. 89, 303-309.

Lee, V.M., Carden, M.J., Schlaepfer, W.W., and Trojanowski, J.Q. (1987). Monoclonal antibodies distinguish several differentially phosphorylated states of the two largest rat neurofilament subunits (NF-H and NF-M) and demonstrate their existence in the normal nervous system of adult rats. $\underline{\mathrm{J} . \mathrm{Neu}-}$ rosci. 7, 3474-3488.

Lighthall, J.W. (1988). Controlled cortical impact: a new experimental brain injury model. J. Neurotrauma $\mathbf{5}, 1-15$.

Marmarou, A., Foda, M.A., Van Den, B.W., Campbell, J., Kita, H., and Demetriadou, K. (1994). A new model of diffuse 


\section{TRAUMATIC AXONAL INJURY IN THE SPINAL CORD EVOKED BY TBI}

brain injury in rats. Part I: Pathophysiology and biomechanics. J. Neurosurg. 80, 291-300.

Maxwell, W.L., Povlishock, J.T., and Graham, D.L. (1997). A mechanistic analysis of nondisruptive axonal injury: a review. J. Neurotrauma 14, 419-440.

Povlishock, J.T. (1993). Pathobiology of traumatically induced axonal injury in animals and man. Ann. Emerg. Med. 22, 980-986.

Povlishock, J.T., and Stone, J. (2001). Traumatic axonal injury, in: Head trauma: basic, preclinical, and clinical directions. H.R. and N.J. Miller (eds), John Wiley and Sons: New York, pps. 281-301.

Povlishock, J.T., Becker, D.P., Cheng, C.L., and Vaughan, G.W. (1983). Axonal change in minor head injury. J. Neuropathol. Exp. Neurol. 42, 225-242.

Povlishock, J.T., Marmarou, A., McIntosh, T., Trojanowski, J.Q., and Moroi, J. (1997). Impact acceleration injury in the rat: evidence for focal axolemmal change and related neurofilament sidearm alteration. J. Neuropathol. Exp. Neurol. 56, 347-359.
Shannon, P., Smith, C.R., Deck, J., Ang, L.C., Ho, M., and Becker, L. (1998). Axonal injury and the neuropathology of shaken baby syndrome. Acta Neuropathol. (Berl.) 95, 625-631.

Sherriff, F.E., Bridges, L.R., Gentleman, S.M., Sivaloganathan, S., and Wilson, S. (1994a). Markers of axonal injury in postmortem human brain. Acta Neuropathol. (Berl.) 88, 433-439.

Sherriff, F.E., Bridges, L.R., and Sivaloganathan, S. (1994b). Early detection of axonal injury after human head trauma using immunocytochemistry for beta-amyloid precursor protein. Acta Neuropathol. (Berl.) 87, 55-62.

Stone, J.R., Walker, S.A., and Povlishock, J.T. (1999). The visualization of a new class of traumatically injured axons through the use of a modified method of microwave antigen retrieval. Acta Neuropathol. (Berl.) 97, 335-345.

Address reprint requests to: Andras Buki, M.D. H-7623 Rét str. 2. Pécs, Hungary

E-mail: 2saturn@gmail.com 